\title{
Estudo da percepção dos benefícios da natação em crianças asmáticas
}

\author{
Study of the perception of the benefits of swimming in asthmatic children
}

Matheus Felipe Mendes

SCARPA

SANDRo Rostelato-Ferreira ${ }^{\mathrm{I}}$

${ }^{1}$ Universidade Paulista (UNIP),

campus Sorocaba/SP-Brasil.

\begin{abstract}
Resumo
Este trabalho tem por objetivo demonstrar a influência da natação na qualidade de vida de crianças diagnosticadas com asma. Foi realizado um levantamento bibliográfico por meio de artigos científicos e revisões, que abordaram o tema da natação para crianças asmáticas. Resultou-se em trabalhos que apontam a natação como sendo um malefício à qualidade de vida das crianças asmáticas, devido à presença de compostos clorados que são utilizados na etapa de desinfecção da água, e tais substâncias se mostram com poder irritativo nas vias aéreas. Por sua vez, dados na literatura demonstram que existem efeitos benéficos em crianças portadoras de asma quando são direcionadas para um programa de natação, resultando em melhora na qualidade de vida, menor tempo de duração nas crises e menor intensidade dos sintomas. Informações recentes de meta-análise concluem que não há diagnóstico aumentado de asma em crianças que praticam a natação. Ancorado nas informações encontradas na literatura, conclui-se que a prática da natação pode ser uma atividade física de baixo impacto para crianças asmáticas, resultando em melhora na qualidade de vida.
\end{abstract}

Palavras-chave: Atividade aquática. Doença respiratória. QUALIDADE DE VIDA.

Abstract The present study aims to demonstrate the influence of swimming on the quality of life of children diagnosed with asthma. A bibliographic survey was carried out through unpublished scientific articles and reviews that addressed the theme of swimming for asthmatic children. It has resulted in studies that point to swimming as a harm to the quality of life of asthmatic children, due to the presence of chlorinated compounds that are used in the disinfection of water, and these substances present with irritative power in the airways. On the other hand, data in the literature show that there are beneficial effects in children with asthma when they are directed to a swimming program, resulting in improvement in quality of life, less durability in the crises and lower intensity of symptoms. Recent meta-analysis concludes that there is no increased diagnosis of asthma in children who practice swimming. Based on the information found in the literature, it is concluded that the practice of swimming can be a low-impact physical activity for asthmatic children, resulting in an improvement in the quality of life.

Keywords: Aquatic activity. Respiratory Disease. QUality of LIFE. 


\section{INTRODUÇÃO}

Asma é a quarta causa mais importante de incapacidades que se apresentam em grupos de crianças entre 10 a 14 anos de idade, ${ }^{1}$ predominando no sexo masculino ${ }^{2}$ e sendo causada por problemas decorrentes da poluição ambiental, ${ }^{3}$ alérgenos, cigarro, ${ }^{4}$ entre outros.

Uma crise asmática pode ter duração de horas ou dias, além de possíveis períodos sem sintomas de dias até meses. A dispneia é predominantemente expiratória e decorrente dos fatores já apontados, sendo o broncoespasmo um fator importante no desencadeamento dos sintomas da asma. ${ }^{5}$

A natação é um exercício saudável que é bem tolerado pelos asmáticos por induzir broncoconstrição menos severa do que outras atividades físicas. ${ }^{6}$ Dados obtidos por Wicher et al. ${ }^{7}$ contribuem para as evidências de que a natação tem efeitos benéficos sobre a capacidade pulmonar dos asmáticos, além da observação dos autores de que ambientes bem ventilados induzem menos sintomas da doença.

Dados opostos aos já apresentados determinam que exposição a produtos desinfetantes de água tem demonstrado ser desencadeante de um quadro asmático. ${ }^{8,9,10}$

Mediante dados divergentes encontrados na literatura, se torna necessário levantar informações acerca da influência da natação sobre a qualidade de vida de crianças asmáticas.

A revisão de literatura foi realizada a partir da busca de publicações indexadas nas bases de dados Scielo, Lilacs e Medline, a partir do cruzamento dos termos: asma/ infância/natação e asthma/childhood/swimming, além de conceitos por meio de livros e dados da Organização Mundial da Saúde. A pesquisa foi limitada aos idiomas inglês e português, e artigos utilizados que foram publicados entre os períodos de 1997 a 2017.

\section{REVISÃO DE LITERATURA}

\section{MALEFÍCIOS DA NATAÇÃO PARA CRIANÇAS ASMÁTICAS}

Estudos apontam que a prática de natação na infância em piscinas cloradas pode aumentar os riscos de infecção no trato respiratório inferior, ${ }^{10}$ pois quando o cloro $\left(\mathrm{Cl}_{2}\right)$ é adicionado à água reage para formar hipoclorito $\left(\mathrm{ClO}^{-}\right)$e ácido hipocloroso $(\mathrm{HClO})$, dependendo do pH. Ambas as substâncias formadas podem gerar outros produtos na água, especialmente, compostos de nitrogênio produzidos pelos nadadores (saliva, suor, urina etc.), resultando na formação de cloraminas (mono, di e tricloroaminas). ${ }^{11}$

A tricloroamina é um composto capaz de causar irritação no trato respiratório e facilmente inalada por indivíduos praticantes da natação, ${ }^{12}$ além de sintomas oculares e respiratórios que têm sido relatado por piscineiros expostos à concentração de tricloroamina no ar, superior a $0,5 \mathrm{mg} / \mathrm{cm} .{ }^{3,13} \mathrm{~A}$ Organização Mundial da Saúde recomenda uma concentração máxima de tricloroamina de $0,5 \mathrm{mg} / \mathrm{cm}^{3}$ presente no ambiente de piscinas cobertas. ${ }^{14}$

Voisin et al. ${ }^{10}$ mostraram que a natação durante a infância, tanto em lugares cobertos ou expostos, está associada a um aumento no risco de bronquiolite, sendo independente de fatores determinantes como a exposição ao tabaco ou doenças preexistentes. Os autores apontam que crianças com bronquiolite, apenas as que praticavam natação, apresentaram maior risco de asma e alergias respiratórias nos anos seguintes.

Bernard et al. ${ }^{15}$ sugerem que a natação na infância pode causar alterações nas vias aéreas, predispondo à asma e bronquite recorrente. Dados esses que foram corroborados 
por Nystad et al., ${ }^{16}$ apontando que a natação antes de 2 anos de idade aumenta a prevalência de infecção do trato respiratório inferior.

Portanto, Voisin et al. ${ }^{10}$ não encontraram diferença significativa entre crianças que praticavam natação quando comparado ao risco de asma e alergias. Embora tais autores demonstrem que a natação na infância está associada a um aumento "dose-dependente" no risco de bronquiolite, ou seja, quanto maior a exposição tanto maior o risco. A exposição às piscinas cloradas nessa idade pode aumentar os riscos de asma e alergias respiratórias durante a infância, sugerindo que a prática de natação na infância pode apresentar um impacto a longo prazo na saúde respiratória das crianças.

\section{BENEFÍCIOS DA NATAÇÃO PARA CRIANÇAS ASMÁTICAS}

A prática da natação é representada como a atividade física com menor potencial de desencadear crises de asma, devido à presença do ar inalado ser mais quente e úmido, sendo capaz de induzir menos broncoespasmo. ${ }^{17}$

Wicher et al. ${ }^{7}$ demonstram efeitos benéficos sobre a função pulmonar de asmáticos. Os autores analisaram 61 crianças com asma atópica, separando-os em grupos controle e natação. No grupo de natação, foi percebido uma hiperresponsividade brônquica que foi reduzida nas crianças que praticaram a natação, e sem observação significativa no grupo controle. A prática da natação tem sido descrita como a mais indicada para crianças asmáticas, por apresentar baixa asmogenicidade (grau de broncoconstrição induzida pelo exercício), quando comparada com outras atividades físicas. ${ }^{18}$

Silva et al. ${ }^{19}$ analisaram o equilíbrio postural de adolescentes (média de idade: $13,3 \pm 1,5$ anos) asmáticos antes e após um programa de natação e ginástica respiratória, concluindo que ambas as técnicas induziram uma diminuição das oscilações e uma melhora no equilíbrio postural, demonstrando a eficiência do programa.

Felcar, Marafigo e Schiavenato ${ }^{20}$ avaliaram um grupo de oito crianças com diagnóstico de asma (7 a 10 anos de idade), que receberam tratamento de fisioterapia na piscina e no solo, resultando melhora na capacidade de exercícios e na qualidade de vida quanto à função emocional em crianças asmáticas. Além de resultados obtidos por meio da prática da natação, existem dados na literatura que determinam o aumento no intervalo entre as crises de asma, diminuição na duração, e as crianças adquirem maior resistência com tal prática, evidenciando melhora nos aspectos psicológicos e sociais, além da eficácia na mecânica respiratória, melhora na ventilação pulmonar e reeducação respiratória. ${ }^{21}$

Lucas $^{22}$ realizou um estudo observacional em criança com asma, além de aplicação de questionário para o responsável, e pôde concluir que a prática de natação trouxe benefícios para o indivíduo estudado, melhorando o quadro sintomático acusada por uma redução do número de crises asmáticas, principalmente à noite.

\section{NATAÇÃO PARA ASMÁTICOS}

A prática da natação tem se mostrado um exercício saudável e bem tolerado por indivíduos asmáticos, aumentando a capacidade aeróbia e melhorando a qualidade de vida dos praticantes. ${ }^{6}$

Ancorado nas informações obtidas pela literatura, pode-se agrupar algumas informações relevantes que estão apresentadas na tabela a seguir (Tabela 1), apontando os dados da influência da prática de natação em crianças asmáticas. 
Tabela 1 - Dados encontrados na literatura sobre os benefícios e malefícios da natação para crianças asmáticas

\begin{tabular}{lll}
\hline \multicolumn{1}{c}{ Autores } & \multicolumn{1}{c}{ Metodologia } & \multicolumn{1}{c}{ Resultados } \\
\hline $\begin{array}{l}\text { Jacques e } \\
\text { Silva }{ }^{21}\end{array}$ & $\begin{array}{l}\text { Entrevista com mães de crianças } \\
\text { asmáticas }(n=17)\end{array}$ & $\begin{array}{l}\text { A prática da natação aumentou o intervalo entre as crises, } \\
\text { diminuiu sua duração, e melhora no grau de intensidade } \\
\text { das mesmas; além de melhora nos aspectos psicológicos e } \\
\text { sociais }\end{array}$ \\
\hline
\end{tabular}

\begin{tabular}{ll}
\hline Voisin et al. $^{10}$ & Aplicaram questionário aos pais \\
& de 430 crianças, com idade média \\
& de 5,6 anos, para saber o histórico \\
& de saúde, prática de natação \\
& e possíveis intervenções nos \\
& resultados
\end{tabular}

Obtiveram resultados de que a frequência em piscinas cobertas ou expostas, antes de 2 anos de idade, foi associada a um risco aumentado de bronquiolite, dependente da exposição para ambos os tipos de piscinas. As associações se mantiveram mesmo após a exclusão dos fatores de risco. As crianças que praticaram natação e desenvolveram bronquiolite apresentaram maiores riscos de asma e alergias respiratórias posteriormente

\begin{tabular}{lll}
\hline $\begin{array}{l}\text { Bernard et } \\
\text { al. }{ }^{16}\end{array}$ & $\begin{array}{l}\text { Analisaram a presença sérica de } \\
\text { proteína de célula Clara e IgE, em }\end{array}$ & $\begin{array}{l}\text { Não foi observado diferença significativa entre o grupo } \\
\text { de natação infantil e as demais crianças em relação à }\end{array}$ \\
& $\begin{array}{l}341 \text { crianças com idade média de } \\
\text { presença de IgE sérica. Os autores concluíram que a prática }\end{array}$ \\
& $\begin{array}{l}\text { de natação infantil em piscinas cobertas e cloradas está } \\
\text { associada a alterações nas vias aéreas que, com outros } \\
\text { fatores, parecem predispor as crianças ao desenvolvimento } \\
\text { de asma e bronquite recorrente }\end{array}$
\end{tabular}

\begin{tabular}{lll}
\hline & & \\
\hline $\begin{array}{l}\text { Andersson } \\
\text { et al. }{ }^{4}\end{array}$ & Questionário (ISAAC) aplicado & $\begin{array}{l}\text { Encontraram um aumento no risco de asma em crianças } \\
\text { sensibilizadas que praticavam natação em piscina coberta, }\end{array}$ \\
& $\begin{array}{l}\text { entre 11-12 anos de idade (n= } \\
\text { 1866) }\end{array}$ & $\begin{array}{l}\text { uma ou mais vezes por semana. Os autores propuseram } \\
\text { uma ligação entre a prática de natação em piscina coberta e } \\
\end{array}$ \\
& asma em crianças sensíveis \\
\hline
\end{tabular}

\begin{tabular}{lll}
\hline Fiks et al. ${ }^{23}$ & $\begin{array}{l}\text { Questionário (ISAAC) aplicado } \\
\text { para crianças (ou responsáveis) }\end{array}$ & $\begin{array}{l}\text { Observaram que a frequência na redução da função } \\
\text { pulmonar foi mais acentuada entre os nadadores que } \\
\text { apresentavam diagnóstico prévio de asma, mas não houve } \\
\text { portade média de } 11 \pm 1,8 \text { anos, } \\
\text { diferença quando comparada com os nadadores não- } \\
\text { de asma e atividade em piscina } \\
\text { clorada }\end{array}$ \\
\hline
\end{tabular}

\begin{tabular}{ll}
\hline Silva $^{24}$ & Estudo realizado em 10 crianças \\
& asmáticas, com idade média de 10 \\
& anos, por meio de um programa de \\
& natação e atividade respiratória
\end{tabular}

Foi apontado diferença significativa após o programa em se tratando da PEmax $(p=0,01)$. Concluiu-se que o programa de natação e ginástica respiratória provocou uma diminuição da ativação da musculatura acessória da respiração e melhora da PEmáx, demonstrando a eficiência do programa

\begin{aligned} \hline Pereira et al. $^{25} &$ Estudaram 42 crianças, entre \\ & meninos e meninas com \\ & idade média de $13 \pm 3$ anos, \\ & diagnosticados com asma, \\ & analisaram os fatores motivacionais \\ & para a prática de atividade física \end{aligned}

Observaram que o fator saúde foi a categoria apontada por $74,36 \%$ das crianças como muito importante, seguida pela dimensão amizade/lazer apontada por $56,41 \%$ também como muito importante. Os autores concluíram que um ambiente de pratica da natação que propicie vivências motoras para a promoção da saúde e não à competição e ao desempenho esportivo é apontado como ideal para crianças e adolescentes asmáticos
IgE - Imunoglobulina E; ISAAC - International Study of Asthma and Allergies in Childhood; PEmax - pressão respiratória máxima




\section{DIscussão}

A prática da natação é um exercício bem tolerado por asmáticos e se considera que a alta umidade do ar inspirado, no nível da água reduz a perda de água pela respiração e possivelmente diminui a osmolaridade do muco das vias aéreas. Assim, a natação é recomendada aos asmáticos com frequência como um meio seguro e agradável de manter a função pulmonar, aumentar sua capacidade aeróbica e melhorar sua qualidade de vida ${ }^{6}$

Por sua vez, existem evidências de que o cloro usado para desinfetar as piscinas seja prejudicial às vias aéreas. ${ }^{16}$ Essa interpretação é apoiada por estudos de biomarcadores que demonstram que os produtos para cloroformização podem causar ruptura crônica ou aguda das barreiras epiteliais dos pulmões. ${ }^{9}$

A prática da natação em piscinas com água não cloradas ou com aumento da ventilação nos ambientes deve ser estimulada, pois os dados apresentados na literatura apontam que ambientes fechados tendem a provocar mais sintomas de irritação nos nadadores, e quando os ambientes estão abertos, os sintomas são reduzidos. ${ }^{26}$

Valeriani et al. ${ }^{27}$ realizaram uma revisão de meta-análise incluindo 2.928 referências (envolvendo 5.821 indivíduos); tal revisão apresentou falha em revelar uma diferença significativa no desenvolvimento de asma em crianças que praticam natação em piscinas expostas a produtos de desinfecção e o grupo controle (Odds Ratio= 1,084 ; faixa de prevalência: 0,58 a 2,30). Os autores puderam concluir que a prática de natação na infância não aumenta o diagnóstico de asma, e a associação da doença com piscinas cobertas ainda é inconsistente.

Embora existam discrepâncias entre os resultados que relatam melhora da função pulmonar em asmáticos e os que apresentam aumento do risco de asma devido piscinas cloradas, os efeitos podem ser resultados que se desenvolvem durante diferentes períodos e provavelmente sob distintas condições de exposição. Contudo, o uso da piscina por tempo superior a 100 horas mostra que o risco de asma aumenta por causa de uma frequência cumulativa que excede a duração do treinamento da maioria dos estudos sobre asmáticos. ${ }^{6} \mathrm{O}$ autor sugere que, em consequência dessas discrepâncias ainda existentes, os nadadores evitem piscinas mal gerenciadas.

Portanto, o treinamento de natação tem se mostrado bem tolerado por crianças e adolescentes com asma estável, apresentando aumento da função pulmonar e da condição cardiopulmonar. Além do mais, não tem sido mostrado evidências de que esse tipo de exercício possa causar efeitos adversos no controle da asma. ${ }^{28}$

\section{CONSIDERAÇões FINAIS}

Ancorado nas informações encontradas na literatura, conclui-se que a prática da natação pode ser uma atividade física capaz de apresentar resultados positivos para crianças asmáticas, resultando em melhora na qualidade de vida, mas que se deve ter cautela quanto ao ambiente em que se realiza a natação, condições clínicas da criança, evitando assim, o desconforto causado pela doença. 


\section{REFERÊNCIAS}

1. Gore FM, Bloem PJ, Patton GC, Ferguson J., Joseph V., Coffey C, et al. Global burden of disease in young people aged 10-24 years: a systematic analysis. Lancet. 2011; 377:2093-102.

2. WHO - World Health Organization. [internet database].10 facts on asthma. <http://www.who. int/features/factfiles/asthma/asthma_facts/en/index.html>, Acesso em: 18/08/2017.

3. McConnell R., Islam T., Shankardass K., Jerrett M., Lurmann F., Gililand F., et al. Childhood incidente asthma and traffic-related air pollution at home and school. Environ Health Perspective, 2010 ; 118: 1.021-6.

4. Andersson M., Modig L., Hedman L., Forsbeg B., Ronmark E. Heavy vehicle traffic is related to wheeze among schoolchildren: a population-based study in an area with low traffic flows. Environ Health, 2011; 13; 10: 91.

5. Teixeira LR, Teixeira AL, Freudenheim AM: Curso de Extensão Universitária em Educação Escolar: Alterações posturais e respiratórias na infância e adolescência, São Paulo, 1991.

6. Bernard A. Asma e natação: pesando os benefícios e os riscos. Jornal de Pediatria, 2010; 86: 5, 351-52.

7. Wicher IB, Ribeiro MA, Marmo DB, Santos CI, Toro AA, Mendes RT, et al. Effects of swimming on spirometric parameters and bronchial hyperresponsiveness in children and adolescents with moderate persistent atopic asthma. J Pediatr (Rio J), 2010; 86 (5): 384-90.

8. Bernard A., Nickmilder M., Voisin C., Sardella A. Impact of chlorinated swimming pool attendance on the respiratory health of adolescents. Pediatrics, 2009; 124: 1.110-8.

9. Bernard A., Carbonnelle S., de Burbure C., Michel O., Nickmilder M. Chlorinated pool attendance, atopy, and the risk of the asthma during childhood. Environ Health Perspec, 2006; 114: 1.567-73.

10. Voisin S., Sardella A., Marcucci F., Bernard A. Infant swimming in chlorinated pools and the risks of bronchiolitis, asthma and allergy. Eur Resp J., 2010; 36: 41-7.

11. Seis SV, Feyen L., Keirsbilck S., Adams E., Dupont LJ, Nemery B. An outbreak of swimming-pool related respiratory symptoms: An elusive source of trichloramine in a municipal indoor summing pool. International Journal of Hygiene and Environmental Health, 2015; 218: 386-391.

12. Catto C., Sabrina S., Ginette CT, Manuel R., Robert T. Occurrence and spatial and temporal variations of disinfection by-products in the water and air of two indoor swimming pools. Int J Environ Res Public Health, 2012; 9 (8): 2.562-2.586.

13. Fantuzzi G., Righi E., Predieri G., Giacobazzi P., Petra B., Aggazzotti G. Airborne trichloramine $(\mathrm{NCl} 3)$ levels and self-reported health symptoms in indoor swimming pool workers: dose-response relationships. J Exposure Sci Environ Epidemiol, 2013; 23: 88-93.

14. World Health Organization. Guidelines for safe recreational water environments. World Health Organization, 2006, p. 2.

15. Bernard A., Carbonnelle S., Dumont X., et al. Infant swimming practice, pulmonary epithelium integrity, and the risk of allergic and respiratory diseases later in childhood. Pediatrics, 2007; 119: 1.095-1.103.

16. Nystad W., Haberg SE, London SJ, et al. Baby swimming and respiratory health. Acta Paediatr., 2008; 97: 657-662.

17. Rund DA: Asthma. The Physician and Sportsmedicine, 1990; 18: 1, 143-146.

18. Oliveira PR. Natação terapêutica para asmáticos. São Paulo: Phorte, 2011.

19. Silva MCR, Silveira MC, Soares JC, Corazza ST, Mota CB. Efeito de um programa de natação e ginástica respiratória no equilíbrio postural de adolescentes asmáticos. R Bras Ci e Mov., 2015; 23 (2): 123-129. 
20. Felcar JM, Marafigo CGA, Schiavenato AC, Torezan RP. Efeitos da associação da hidroterapia e exercícios em solo em crianças asmáticas. In: 13․ Simpósio Internacional de Fisioterapia Respiratória e Fisioterapia em Terapia Intensiva, 2006, Curitiba, Revista Brasileira de Fisioterapia, 2006; 1: 19-19.

21. Jacques GP, Silva OJ. Influência da natação como coadjuvante terapêutico no tratamento de crianças asmáticas. Rev Bras Med Esport., 1997; 3: 1,15-21.

22. Lucas MSR. Os benefícios da natação para crianças com asma. Monografia (Graduação) Fundação Universidade Federal de Rondônia. UNIR, Porto Velho, 2015.

23. Fiks IA, Santos LCA, Antunes T., Gonçalves RC, Carvalho CRF, Carvalho CRR. Frequência de sintomas de asma e de redução da função pulmonar entre crianças e adolescentes nadadores amadores. J Bras Pneumol., 2009; 35 (3): 206-212.

24. Silva MCR. Efeito de um programa de natação e ginástica respiratória sobre a ativação de músculos acessórios da respiração em asmáticos. Dissertação (Mestrado em Educação Física), 67s. Universidade Federal de Santa Maria, RS, 2014.

25. Pereira EF, Teixeira CS, Villis JMC, Paim MCC, Sanchotene L., Daronco E. Fatores motivacionais de crianças e adolescentes asmáticos para a prática da natação. R Bras Ci e Mov., 2009; 17 (3): 9-17.

26. Wicher IB, Ribeiro MAG, Marmo DB, Santos CIS, Contrera AAD, Mendes RT, Cielo FMBL, Ribeiro JD. Effects of swimming on spirometric parameters and bronchial hyperresponsiveness in children and adolescents with moderate persistent atopic asthma. Jornal de Pediatria, 2010; 86: 5,384-390.

27. Valeriani F., Protano C., Vitali M., Romano Spica V. Swimming attendance during childhood and development of asthma: a meta-analysis. Pediatr Int., 2017; 59 (5): 614-621.

28. Beggs S., Foong YC, Le HCT, Noor D, Wood-Baker R., Walters JAE. Swimming training for asthma in children and adolescents aged18 years and under. Cochrane Database of Systematic Reviews, 2013, Issue 4. Art. No.: CD009607.

\section{DAdos dos AUTORES}

\section{Matheus Felipe Mendes Scarpa}

Graduado em Educação Física pela Universidade Paulista (UNIP). Professor de natação em academia. Salto de Pirapora/SP - Brasil. matheuscarpa@hotmail.com

\section{Sandro Rostelato Ferreira}

Graduado em Farmácia Bioquímica pela Universidade de Sorocaba (UNISO), Mestre e Doutor em Farmacologia pela Universidade Estadual de Campinas (UNICAMP). Professor na Universidade Paulista (UNIP). Sorocaba/SP - Brasil. sandrorostelato@yahoo.com.br

Submetido em: 12-9-2017

Aceito em: $14-2-2018$ 